\title{
Jacques Grand’Maison
}

Sociologue, Université de Montréal

(2003)

\section{"Grandeur et misère
de la parentalité moderne"}

Un document produit en version numérique par Jean-Marie Tremblay, bénévole, Professeur sociologie au Cégep de Chicoutimi

Courriel: jean-marie_tremblay@uqac.ca

Dans le cadre de "Les classiques des sciences sociales"

Site web: http://classiques.uqac.ca/

Une bibliothèque fondée et dirigée par Jean-Marie Tremblay, sociologue

Une collection développée en collaboration avec la Bibliothèque

Paul-Émile-Boulet de l'Université du Québec à Chicoutimi

Site web: http://bibliotheque.uqac.ca/ 
Cette édition électronique a été réalisée Jean-Marie Tremblay, bénévole, professeur de soins infirmiers retraitée de l'enseignement au Cégep de Chicoutimi

Courriel: jean-marie_tremblay@uqac.ca

à partir du texte de la conférence de :

Jacques Grand’Maison, “Grandeur et misère de la parentalité moderne”. Texte d'une conférence prononcée à l'occasion de la journée d'étude "Nos enfants ont droit à leurs deux parents", tenue le 14 novembre 2003 au Centre St-Pierre.

M. Jacques Grand'Maison (1931 - ) est sociologue (retraité de l’enseignement) de l'Université de Montréal.

[Autorisation formelle réitérée le 6 mars 2004 au téléphone par M. Jacques Grand’Maison et confirmée par écrit le 15 mars 2004 de diffuser la totalité de ses œuvres : articles et livres]

S4 Courriel : diocesesj@citenet.net

Polices de caractères utilisée :

Pour le texte: Times New Roman, 14 points.

Pour les citations : Times New Roman 12 points.

Pour les notes de bas de page : Times New Roman, 12 points.

Édition électronique réalisée avec le traitement de textes Microsoft Word 2004 pour Macintosh.

Mise en page sur papier format : LETTRE (US letter), 8.5’’ x 11’’)

Édition numérique réalisée le 8 mai 2006 à Chicoutimi, Ville de Saguenay, province de Québec, Canada. 


\section{Table des matières}

Présentation de Jacques Grand'Maison

À propos de cette conférence

$\underline{\text { Texte de la conférence de M. Grand'Maison }}$

Le refus de la limite.

L'indifférenciation.

La désinstitutionalisation.

Les atouts et défis d'avenir.

Les enjeux de la paternité et de la filiation.

Un positionnement d'espérant têtu. 
Jacques Grand'Maison, “Grandeur et misère de la parentalité moderne”.

Texte d'une conférence prononcée à l'occasion de la journée d'étude "Nos enfants ont droit à leurs deux parents", tenue le 14 novembre 2003 au Centre StPierre.

http://cf.geocities.com/francaislangueseconde/grandeur_et_misere_de_la_parentalite_moderne.htm

\section{Présentation de Jacques Grand'Maison}

$\underline{\text { Retour à la table des matières }}$

Jacques Grand'Maison est à la fois sociologue et théologien. Il fit ses études au Grand séminaire de Montréal, à l'Université Grégorienne au Vatican et obtint son doctorat à l'Université de Montréal où il fut professeur titulaire à la Faculté de théologie de 1967 à 1997.

QuickTime $^{\mathrm{TM}}$ and a TIFF (non compressé) decompressor are needed to see this picture. 
Par sa double formation académique, il a démontré la nécessité de décloisonner les discours enfermés dans les délimitations disciplinaires, que ce soit celles du droit, de la sociologie, de la psychologie ou de la théologie.

Auteur de plus de 40 ouvrages, il a collaboré de façon déterminante à plus d'une vingtaine d'autres. Sa plus récente contribution au débat entourant l'évolution de la société québécoise s’exprime dans un petit réquisitoire intitulé "Questions interdites sur le Québec contemporain" paru Aux Éditions Fides

\section{À propos de cette conférence}

$\underline{\text { Retour à la table des matières }}$

«C'est à titre d'éducateur depuis 50 ans que je vais traiter de la mission éducative fondamentale de la parentalité. Avec une attention particulière au socle majeur de l'altérité des pôles masculin et féminin. Je vais situer ma réflexion dans notre propre contexte de société qui façonne, lui aussi, par ses tendances dominantes les traits marquants d'une personnalité de base qu'on peut trouver dans tous les milieux sociaux . Cet exposé comportera un volet critique et un volet dynamique. » 


\section{TEXTE DE LA CONFÉRENCE}

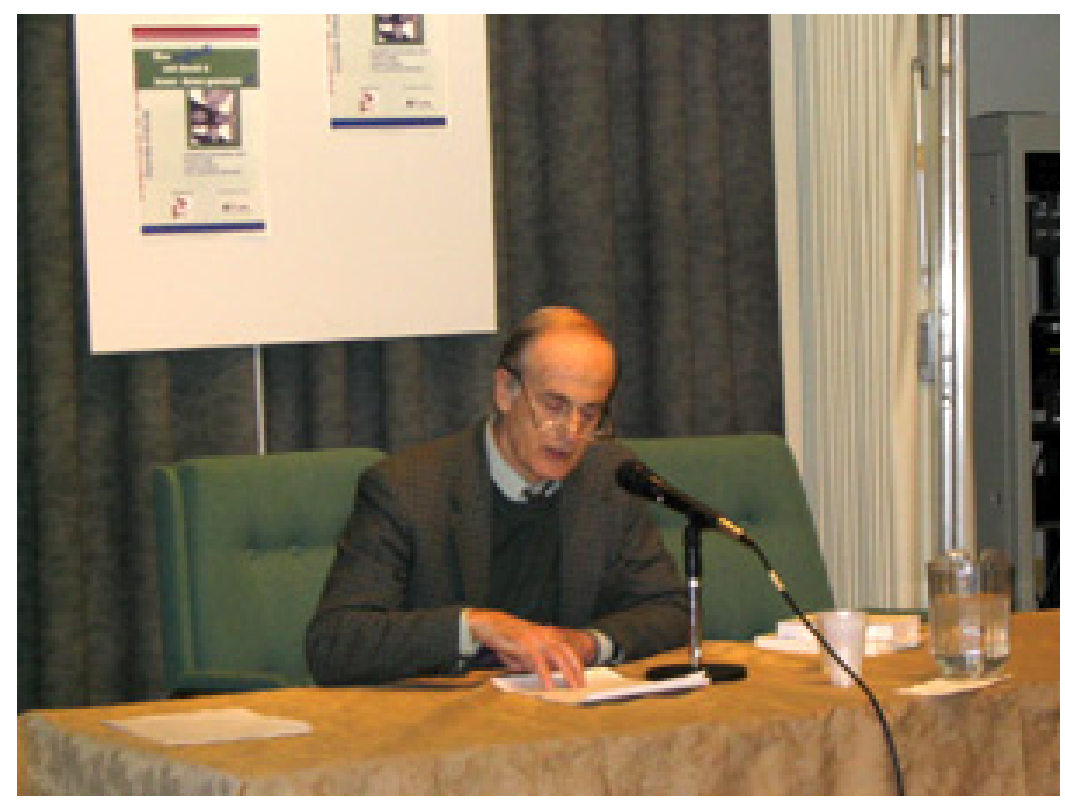

$\underline{\text { Retour à la table des matières }}$

Permettez-moi d'abord de me situer personnellement dans ce colloque et sa thématique pour circonscrire à la fois les limites et le champ singulier de vision de mon exposé.

Depuis un bon moment, je travaille sur la personnalité de base que génèrent notre société et les courants sociaux et culturels dominants qui la traversent de part en part, et qui retentissent dans ses rapports fondamentaux, et cela jusque dans la conscience des individus, avec une attention particulière à la famille, à l'éducation. Depuis cinquante ans, comme éducateur, j'ai accompagné les jeunes générations qui se sont succédées et j'ai suivi leurs itinéraires d'adultes, de parents et de citoyens. Et cela à travers des projets collectifs auxquels j'ai été asso- 
cié. Je tenais à ces ancrages concrets qui me permettaient de conjuguer théorie et pratique; recherche, action et formation.

Je ne vous dis pas cela pour lever mon petit drapeau, mais pour marquer l'axe majeur de mon exposé dont la dominante sera une approche, un regard, un positionnement d'éducateur. En même temps, je marque ainsi mes limites. J'ai éduqué les enfants des autres. Je n'ai pas moi-même une expérience de parent, ni de couple; ce que vous avez ici pour la plupart. Par ailleurs, je fais le pari que cette distance peut être utile, comme peut l'être celle du sociologue que je suis. La famille, vous en conviendrez, est tributaire de l'évolution de la société. Vous savez, comme parents, que bien d'autres influences que la vôtre jouent sur vos enfants. Ce qui m'a permis d'éviter de prendre les parents comme boucs émissaires de tous les problèmes des jeunes d'hier ou d'aujourd'hui.

Mon exposé comporte un versant critique et un versant plus positif et dynamique. Je tiens d'abord à souligner qu'il y a eu au cours des dernières décennies plusieurs changements bénéfiques dans la famille moderne. Mais depuis quelques temps ont émergé certains travers et dérives graves qui concernent la thématique de ce colloque.

Je retiens, sur le versant critique, trois courants qui ont marqué à la fois la société et ce qu'on appelle en psychosociologie la personnalité de base. Je vais aborder ces trois courants en relation avec leurs impacts sur la famille, sur la parentalité, sur les jeunes eux-mêmes. Dans mes études en sciences sociales, et particulièrement en anthropologie, j'ai appris que les rapports hommes-femmes et les rapports de générations sont des assises plus profondes que tous les autres rapports sociaux, économiques ou politiques. Il en va de même des différents rôles que se donne un système social. La définition des rôles dans la famille a une spécificité, un caractère unique par rapport aux rôles dans les autres institutions. Dans la famille, comme le dit si bien Louis Roussel dans son ouvrage La famille incertaine: L'enfant reçoit des mêmes personnes la possibilité de réaliser ses désirs et l'ordre de les limiter. Cela fait de la famille une institution unique dans la société. Et nous verrons les graves conséquences qui adviennent quand l'un ou l'autre de ces deux apprentissages de base est déficient, non seulement pour sa vie d'enfant et sa vie future mais aussi pour ses rapports aux 
autres, à son travail et à la société comme futur citoyen. Il en va de même de l'influence inverse quand, par exemple, la société transpose dans la famille une logique juridique de l'individu insulaire, mesure de toutes choses. Renée Joyal a bien montré les effets pervers de la désinstitutionalisation de la famille, de ses rôles et des rapports hommesfemmes, père-mère, parents-enfants.

Ce qui m'amène à souligner les trois courants annoncés plus haut et à les expliciter.

- Le premier est le refus de la limite

- Le deuxième est l'indifférenciation

- Le troisième est la désinstitutionalisation

Trois courants plus ou moins souterrains, peu explorés, peu reconnus, et même objet d'interdits parce qu'ils remettent en cause la culture narcissique dominante, la pop-psychologie dont nous sommes saturés, et cela jusqu'aux politiques des gouvernements et les logiques juridiques qui prévalent. Beaucoup de revendications et de débats actuels de tous ordres marquent même un refus de prendre en compte ces trois tendances et les pratiques qui les accompagnent. Cet enjeu ne se joue pas seulement au Québec ou au Canada mais dans toute l'Amérique du Nord, comme en témoigne Christopher Lasch dans son dernier ouvrage dont le titre est ironique: "Le seul et vrai paradis". Selon lui, nous sommes dans une société passoire où on peut et on veut justifier à peu près n'importe quoi et où toute résistance à cette dite ouverture illimitée est stigmatisée par les médias, par les dites élites et la pression publique. On peut contester l'exagération de ce diagnostic qui laisse entendre que la seule normalité qui reste est celle de n'en avoir aucune. Et l'on se plaint en même temps de l'absence de repères stables chez les jeunes. On préfère parler vaguement de meilleur encadrement ou d'environnement plus favorable. Par exemple pour surmonter les difficultés actuelles des garçons.

Les trois courants dont j'ai parlé plus haut n'ont rien d'abstrait. Ils ont une histoire concrète dans l'évolution récente de la société. 
Le refus de la limite.

Le refus de la limite, on le trouve déjà chez le Docteur Spock dans les années ' 50 . Et plus largement dans les trois mythes qui ont dominé depuis 50 ans en Occident: à savoir une croissance économique illimitée, un État Providence illimité et une libéralisation des mœurs illimitée avec trois certitudes: on peut tout prévoir, tout faire et tout se permettre. À cela s'ajoute une culture narcissique du "déjà tout en toi", de l'enfant-roi, de l'adulte adulescent, de nombre d'aînés bien portants sans âge qui réclament des privilèges de l'enfance: divertissement permanent et satisfaction illimitée. Sans compter le mythe nordaméricain le plus écrasant de tous, mythe qui commande d'être à la fois et pour toujours: beau, jeune, riche, en parfaite forme et libre de tout lien, de toute contrainte. En combien d'autres domaines ne sombre-t-on pas dans la démesure, dans l'extrême, dans l'illimité. Tout le Québec pâmé devant Star Académie qui prétend fabriquer des grandes vedettes en quelques semaines. Peut-on imaginer un message aussi anti-éducatif qui ne souffre aucune distance critique et même la moindre réserve. Encore moins, n'y a-t-on vu l'aboutissement de la bonne douzaine d'utopies qu'a véhiculées le monde de l'éducation depuis cinquante ans. Pensons à la plus effarante de toutes, résumée par ces slogans: "Personne ne transmet rien à personne".

Voilà un premier fond de scène critique derrière la problématique spécifiquement familiale et parentale de Louis Roussel sur les deux apprentissages de base: permettre à l'enfant de réaliser ses désirs et l'ordre de les limiter. Pas facile d'éduquer un enfant quand de toutes parts on sombre dans l'illimité.

On a fait une étude très éclairante au Danemark sur les effets pervers de la démission de l'autorité parentale dans le psychisme de l'enfant. On s'est rendu compte que l'enfant privé de limite était hanté dans son inconscient par un père illimité, écrasant, fantasmé, ou par 
une super mère monoparentale elle-même fantasmée et aussi écrasante. Je tire cela d'un ouvrage d'Élisabeth Badinter.

\section{L'indifférenciation.}

Le deuxième point critique, celui de l'indifférenciation, est intimement relié au refus parental de la limite. Avez-vous lu ou entendu beaucoup de choses sur la tendance souterraine pourtant fort répandue, celle de nier les différences de sexes, de rôles et de générations. En a-t-il été question dans les débats et la mise en législation de l'homoparentalité, par exemple? Pourtant dans les sciences humaines il y a pourtant consensus sur les processus de différenciation nécessaires à la constitution de l'identité sexuelle, personnelle et sociale du garçon et de la fille, particulièrement sur la différenciation du pôle masculin et du pôle féminin, socle aussi bien existentiel que symbolique de toutes les autres altérités.

Encore ici, vous allez trouver des tendances à l'indifférenciation qui traversent toute la société et la culture. Combien de modes psychologiques et religieuses tiennent de l'indifférenciation malgré tout ce qu'on dit sur l'autonomie, sur l'individualité. Les rôles sont interchangeables. Comme dit Blanche Tahon: "on attend du père qu'il soit une seconde mère". Dans notre étude sur l'univers intérieur des jeunes suicidaires, on s'est rendu compte que leur univers intérieur était indifférencié sans repères, sans limite. Ne sont-ils pas dans une société ou tout est égal?" Comme disait un adolescent à son père: "papa, des copains j'en ai en masse, un père je n'en ai qu'un". Ou cette fille de 16 ans qui disait à la psychologue de son école: "Ma plus grande souffrance, c'est qu'il n'y a personne au-dessus de moi". Ou cette autre fille accompagnée de sa mère qui voulait à tout prix être présente à la rencontre avec le psychologue sous ce prétexte: "Moi et ma fille, on se dit tout." Le psychologue insiste pour d'abord rencontrer sa fille seule. Et celle-ci de dire au psychologue: "C'est justement ça mon problème, ma mère ne cesse de me parler de ses problèmes à elle, ses problèmes 
avec son nouvel amant, mais moi j'en ai déjà assez de mes propres problèmes".

Et oui, tout est égal. Confusion des rôles, des âges, des générations, fi des différences de rôles différents du père et de la mère, car ce sont des préjugés sexistes. Fussent ces acquis de la psychologie: tels le caring de la mère et le rôle du père qui amène l'enfant hors du cocon familial. Un autre préjugé sexiste!

On ne compte plus les pratiques d'indifférenciation et du tout est égal et des solutions mur à mur. Autrefois on mêlait la religion à tout. Aujourd'hui la dernière mode au ministère de l'éducation c'est de demander à tous les acteurs de l'école d'aborder les questions de sexualité dans toutes les matières avec cette incroyable légitimation, à savoir qu'on est dans une société hyper sexualisée. Encore fi de ce que nous a appris la psychologie, à savoir ceci: l'enfant de 8-9-10-11-12 ans vit une période de latence de sa sexualité pour s'ouvrir à d'autres dimensions et intérêts au-delà du cocon affectif et sexué familial. Voilà un autre processus de différenciation dans la construction personnelle et sociale de l'enfant et son inscription progressive dans le monde réel et la société dont l'école est une médiation importante de socialisation.

Autre source d'indifférenciation, celle-là plus paradoxale à savoir la transposition à tous les âges de la vie de la condition infantile. Par exemple, comme c'est le cas de la carte unique qu'on est en train de jouer à l'école secondaire. La carte du plaisir en tout et partout. J'ai vu un reportage à la télé qui exaltait cette politique pédagogique, avec aucune distance critique et une ignorance crasse des longues et sérieuses études qui ont été faites sur cela. Je pense à celle de Bruno Betheleim. Celui-ci a constaté que la carte du plaisir était efficace à court terme, mais à moyen terme et à long terme le jeune décroche quand il n'a plus de fun; il n'a pas alors d'autres cordes de motivation pour poursuivre ses études. Voilà un autre indifférenciation. 


\section{La désinstitutionalisation.}

Le troisième point critique est celui de la désinstitutionalisation, lui aussi relié aux deux autres. Une de vos propres personnes ressources, la juriste Renée Joyale, a montré comment depuis une quarantaine d'années la famille a été désinstituée. J'espère ne pas trop trahir la pensée en rapportant son propos à ce chapitre. La famille s'est toujours définie jusqu'à présent, selon une représentation bipolaire. Il s'agit aussi d'une institution généalogique qui institue la possibilité d'une certaine durée dans les relations humaines et les rapports sociaux. Or, en présentant la famille comme une configuration institutionnelle contingente, sujette à démembrement, à partir de la figure du contrat et en dehors de toutes considérations normatives, c'est aux fondements même de la famille qu'on s'en prend plus ou moins directement. C'est alors la toute puissance des désirs et de l'intérêt immédiat qui prend le dessus, et le droit, plutôt de traduire des normes et repères institutionnels normalement de nature stable, doit tout au plus se présenter comme une régulation à géométrie variable. Il ne s'agit pas ici de changements mineurs, si tant est qu'on ait compris que la famille comme institution de la vie humaine est un socle très important de la société. Plus largement on se rappellera que les institutions régulent le vivre-ensemble et l'agir ensemble dans l'un ou l'autre champ de la vie collective avec un souci de viabilité commune et d'inscription dans le temps. Ce qui est en cause dans certaines dérives récentes, c'est l'effacement du sens même de ce qu'est une institution. Les débats récents sur le mariage et la parentalité ont été projetés dans le procès de la religion, alors que la question de fond est laïque et anthropologique, même avant toute considération morale.

Voyons cela de plus près, avec des exemples concrets à partir de l'image métaphore du self service en matière de droit et des droits. Récemment une petite fille de 11 ans a été traitée comme un sujet juridique adulte pour obtenir légalement que son père n'ait plus accès à 
elle. Le dossier judiciaire révélait que l'enfant avait eu de très bons rapports avec son père avant le divorce de ses parents et que la mère avait tout fait pour que le père n'ait pas accès à sa fille. Ce que je retiens le plus, c'est la démarche du juge qui a traité l'enfant comme un adulte avec des critères subjectifs: "cet enfant, dit le magistrat, est étonnamment mature et plus intelligente que la moyenne." Ce cas n'est qu'un exemple d'une pseudo-logique juridique qui réduit le droit à celui de l'individu insulaire et même à son unique subjectivité, sans les liens sociaux et en l'occurrence, familiaux, inhérents au droit. Ce qui contredit le sens premier de ce qu'est une institution dont un des rôles fondamentaux est la régulation du lien social.

Il en va de même d'un problème de plus en plus fréquent, celui de grossesses d'adolescentes où l'on peut légalement procéder à un avortement à l'insu des parents. Si de graves problèmes psychologiques surviennent chez l'adolescente après l'avortement, comment ses parents peuvent-ils l'aider s'ils ne savent rien de ce qui s'est passé. Ce sont les parents qui ont à faire face quotidiennement à ces problèmes et non les tiers intervenants. Un autre exemple de désinstitutionalisation. On sait depuis toujours que la paternité est passablement abstraite en regard de la maternité qui met au monde garçons et filles. Pour éveiller le père à une paternité plus consciente, plus responsable, la société occidentale a institué des déterminations symboliques, par exemple, donner son nom à l'enfant. Voyez ce qu'on a fait depuis quelques années à ce chapitre.

Je pourrais allonger la liste de ces désinstitutionalisations de la famille. Dans les événements récents, surtout à propos du mariage des homosexuels et de l'homoparentalité, j'ai été étonné du peu de place qu'on a donnée aux enjeux de la filiation. Toutes les sciences humaines comme je le disais plus haut, s'entendent sur l'importance des pôles masculin et féminin dans la constitution de l'identité personnelle, sexuelle et sociale du garçon et de la fille. D'où vient donc le silence sur cette donnée importante dans les milieux professionnels et scientifiques?

Voilà pour ce versant critique mais il y a aussi un versant positif dans ce qui nous arrive. Derrière ce qui se défait souvent des pousses nouvelles surgissent. 
Les atouts et défis d'avenir.

Retour à la table des matières

Il y a présentement une revalorisation de la filiation, qui est un des rares lieux et liens humains durables dans une société où tout se joue à court terme dans pratiquement tous les domaines. On peut dire son exconjoint, mais on peut pas dire son ex-enfant. La filiation n'est-elle pas la première base de la conscience historique et de l'inscription dans le temps? La majorité des adultes de la planète trouvent dans leurs enfants la plus forte motivation au dépassement, au courage, à l'engagement et à la responsabilité de leur offrir une société plus humaine et plus heureuse. Nous sommes divisés politiquement, culturellement, religieusement, mais il nous reste cette assise commune très existentielle qu'est l'avenir de la jeune génération. Que deviendront nos sociétés vieillissantes sans une jeunesse bien éduquée, heureuse et assez nombreuse. On commence à se rendre compte de cet enjeu crucial.

En regard des sociétés européennes nos politiques proprement familiales marquent un retard à combler. Il faut travailler à ce que les jeunes adultes aient de meilleures conditions pour des projets de famille viables. L'histoire de nos sociétés occidentales au XXe siècle nous a révélé qu'une jeune génération éreintée au départ risque de traîner cela toute sa vie. Encore ici le sort des enfants est le plus grand test de vérité et d'humanité de notre société, de toute société, et aussi de nos attitudes les plus profondes face à la vie, face à l'avenir.

Dans une histoire de plus en plus imprévisible et des sociétés de plus en plus éclatées et instables, la famille malgré tous ses avatars apparaît à plusieurs d'entre nous comme un des premiers et plus importants socles pour retrouver foi en nous-mêmes, en la vie, en l'avenir. Voyons bien l'heureux paradoxe de la situation présente: après avoir décrété que la famille n'est plus la cellule de base de la société, après avoir dissocié le mariage de la filiation, voici que contre toute attente, la majorité des gens, y compris des jeunes redonnent à la fa- 
mille un sens majeur. Il faut que les associations de familles soient plus actives face à la société, face aux médias actuels pour rappeler cet enjeu capital d'avenir, pour que la majorité des enfants puissent se construire sainement avec des hommes et des femmes, des pères et des mères, des adultes qui redonnent à la filiation son importance majeure et mesurent leurs débats, leurs conflits, leurs revendications à l'aune de ce qui arrive aux enfants. Beaucoup de législations actuelles et de débats entre adultes, et même de solutions pour adultes, marginalisent ou laissent trop en veilleuse le sort des enfants. Je le redis: il faut contrer positivement cette tendance mortifère ou la seule normalité qui risque de rester, c'est de n'en avoir aucune.

Une des plus riches caractéristiques de la famille moderne standard ou recomposée est de permettre à chacun de ses membres d'engager sa propre histoire. À ce chapitre la révolution féminine a joué un rôle inestimable. Mais en même temps, les associations de familles peuvent rappeler qu'on peut difficilement engager sa propre histoire personnelle, si à la base de sa vie, la famille est éphémère et sans histoire durable, si la société est construite avant tout sur l'individu insulaire, si on continue de désinstituer la famille, de mille et une façons, si on n'assume pas mieux le malaise masculin actuel. $80 \%$ des suicides sont dans le monde masculin. 4 fois plus de décrocheurs et combien d'autres déficits masculins. Je pense à ce petit garçon de 8 ans qui disait à ses parents: "c'est'y vrai que tous les hommes sont méchants et violents comme y disent à la télé?"

Moi je souhaite que les associations de famille soient plus engagées "citoyennement". Elles ont des choses très importantes à dire à la société actuelle, aux médias et pas seulement aux gouvernements et aux hommes politiques, mais aussi à des spécialistes, à des professionnels et à des idéologues qui dissocient de plus en plus le mariage de la famille et de la filiation. Le silence actuel de ceux qui ne sont pas d'accord avec cela est plus qu'étonnant.

Dans ma première rédaction de l'exposé que j'avais à vous donner aujourd'hui je vous faisais part de pratiques d'implication des parents, des deux parents, mais je me suis rendu compte que j'empiétais sur les ateliers qui allaient suivre. J'ai préféré mettre en perspective le fond de scène de nos échanges d'aujourd'hui. Et dans les limites inévitables 
d'une conférence d'ouverture, j'ai plutôt privilégié certains enjeux cruciaux actuels qui concernent profondément la famille.

Tous les sondages font état de l'importance que les gens accordent à la famille et en même temps on se retrouve avec des tendances, des pratiques, des débats et même des législations qui contredisent l'importance qu'on accorde idéalement ou autrement à la famille. Certains, même parmi les plus sensibles à la cause des enfants soutiennent qu'on n'a pas besoin de politiques familiales, mais plutôt de politiques sociales. À tort ou à raison je pense que cette opposition simpliste révèle implicitement, chez certains, quelle place véritable ils donnent à la famille, et chez d'autres, l'esquive de questions spécifiquement familiales qu'ils ne veulent pas entendre.

Marie-Blanche Tahon disait que les femmes sont encore socialement discriminées, malgré les progrès accomplis, et que les pères, eux, sont discriminés dans la désinstitutionnalisation de la famille.

D'où mes dernières questions: Que ce cachet-t-il derrière le slogan: "Un enfant si je le veux et quand je le veux". Quel message lance-t-on aux hommes et aux pères dans le formulaire d'inscription de l'enfant dans les registres civils actuels quand on leur donne 30 jours pour signifier qu'ils sont bien le père?

Dans un autre ordre de questionnement, se peut-il que certaines attentes équivalent à exiger du père qu'il soit une seconde mère?

C'est un des rôles d'une conférence d'ouverture de poser des questions qu'on n'ose pas poser.

Les enjeux de la paternité et de la filiation.

$\underline{\text { Retour à la table des matières }}$

Étant donné qu'il me reste un peu de temps, j'aimerais dire quelques mots sur les pères. Dès le début de mon travail social, j'ai eu à faire un joli virage dans ma façon d'aborder les parents en relation avec un ou l'autre des problèmes graves de leur jeune. Souvent le père 
était celui qui avait le plus de difficulté à faire face au problème. Il se sentait humilié et impuissant devant cette situation, d'où des réactions fortes ou la fuite. J'ai changé radicalement mon approche comme ceci. Je soulignais d'abord les qualités de leur jeune en soulignant qu'ils étaient pour quelque chose dans ces atouts positifs de leur jeune. L'atmosphère se détendait et l'on pouvait travailler ensemble pour aider le jeune à s'en sortir.

Dans mon équipe de recherche sur les générations et leurs rapports, il y avait un psychologue de 35 ans, père de deux enfants. Il nous a raconté ceci: Vous savez, je suis psychologue, et pourtant quand un de mes propres enfants a un problème qui nous amène à l'école ou au CLSC ou à l'hôpital, je suis porté à m'en remettre à ma femme. Et pourtant, je suis passablement bien équipé pour faire face à cela.

Ces propos nous font soupçonner l'inévitable long chemin à parcourir pour redéfinir et réinscrire les pères dans les tâches éducatives familiales. Les jeux de rôles où le père et le fils inversaient leurs rôles m'ont appris beaucoup de choses, outre les déblocages que ces dramatiques ont suscités. Notre jeune psychologue nous a alertés sur toute la part d'impensé, d'informel qu'il y a dans la conscience masculine aussi bien d'aujourd'hui et d'hier. Une sorte de sous-détermination que l'indifférenciation des rôles dans la parentalité moderne risque de renforcer. Plusieurs pères sont surdéterminés dans leur dénégation: "Je n'ai pas de problème" et sous déterminé dans leur conscience identitaire comme père. Les femmes modernes se sont redéfinies davantage. Je ne suis pas sûr qu'on ait pris la mesure du fait systémique qu'un changement de rôle amène un changement de tous les autres rôles dans la famille. Réduire la structure ternaire de la famille à un seul critère: l'égalité des individus qui s'y trouve. À ce chapitre je suggère la lecture des ouvrages de Louis Roussel, Marie-Blanche Tahon, Pierre Legendre.

Je pense aux trois types de famille moderne bien analysées par Louis Roussel dans son ouvrage: La famille incertaine (Éd. Odile Jacob, 1989). Avec une judicieuse finesse d'analyse il montre ce qui advient de la parentalité et de la filiation dans ces trois types de famille qu'il qualifie ainsi: la famille fusionnelle, la famille club, la famille généalogique. Les rapports à l'enfant sont loin d'être les mêmes dans 
ces différents types de famille. J'insiste sur cela parce que dans tant de tendances et de débats actuels, c'est l'enfant, le plus faible chaînon, qui paie le plus cher la note. La note d'une société et d'une culture dominante où l'individu insulaire devient la mesure de tout, même dans tant d'aventures de couple, dans tant d'éclatements familiaux. Jusqu'à cette utopie mortifère qu'on trouve même chez des philosophes dits postmodernes qui flirtent avec la pop-psychologie de l'auto-enfantement de soi par soi; c'est là la source empoisonnée de la marginalisation de toutes les filiations familiales, historiques et culturelles, de l'ère de l'éphémère et des nombreuses crises de transmission. Je pense, par exemple, à la situation dramatique du bloc opératoire de notre hôpital régional où du jour au lendemain 10 infirmières chevronnées sur 11 sont parties en retraite anticipée sans véritable politique de transmission aux jeunes infirmières qui les ont remplacées.

Du coup on se rend compte de l'importance de bien comprendre l'importance pour toute la société de ce qui arrive dans le premier socle de l'institution de la vie qu'est la famille. C'est en celle-ci que l'idéologie de l'individu insulaire brise la structure ternaire de la famille, de la parentalité et de la filiation.

\section{Un positionnement d'espérant têtu.}

Retour à la table des matières

Je suis conscient de la complexité des propos que je viens de tenir. Mais diable, on ne peut admettre que les défis et les problèmes auxquels nous faisons face sont profonds et souvent compliqués, et en même temps souhaiter des diagnostics et des solutions faciles et prêtes à porter.

Je suis aussi conscient des limites de ma démarche singulière, des matières à débat que j'ai soulevées. Nous sommes condamnés à avoir du génie ensemble. Nous sommes ici pour affronter ce défi qui appelle un consentement à élever notre réflexion de plusieurs crans.

Mon positionnement en est un d'espérant têtu. En acceptant d'affronter les enjeux les plus cruciaux, on est plus susceptible d'aller 
chercher en soi et avec les autres des sources et ressources plus profondes. Malraux définissait l'éducation dans cette foulée dynamique. Il y a chez l'être humain une formidable capacité morale et spirituelle de rebondissement dont l'histoire témoigne. Il est bon de se le rappeler et de l'intégrer pour donner plus de sens à nos tâches et engagements. La génération montante a besoin de cet héritage inestimable qui nous vient d'une longue filiation des générations.

J'ai le sentiment de vous livrer ici le testament du vieil éducateur que je suis, qui, encore aujourd'hui, donne priorité à l'avenir de la jeune génération.

Dans la foulée de cette passion de ma vie je suis hanté par la question qui est au cœur de toutes les formes de parentalité: Qu'est-ce que nous transmettons au juste aux générations qui nous suivent? Voilà une autre question trop laissée pour compte. Fera-t-elle partie de ce colloque qui concerne en tout premier lieu: la filiation, toutes les filiations. Le pire drame humain et spirituel d'un jeune, c'est d'être entouré d'adultes qui ne croient plus en grand chose.

À bon entendeur, salut!

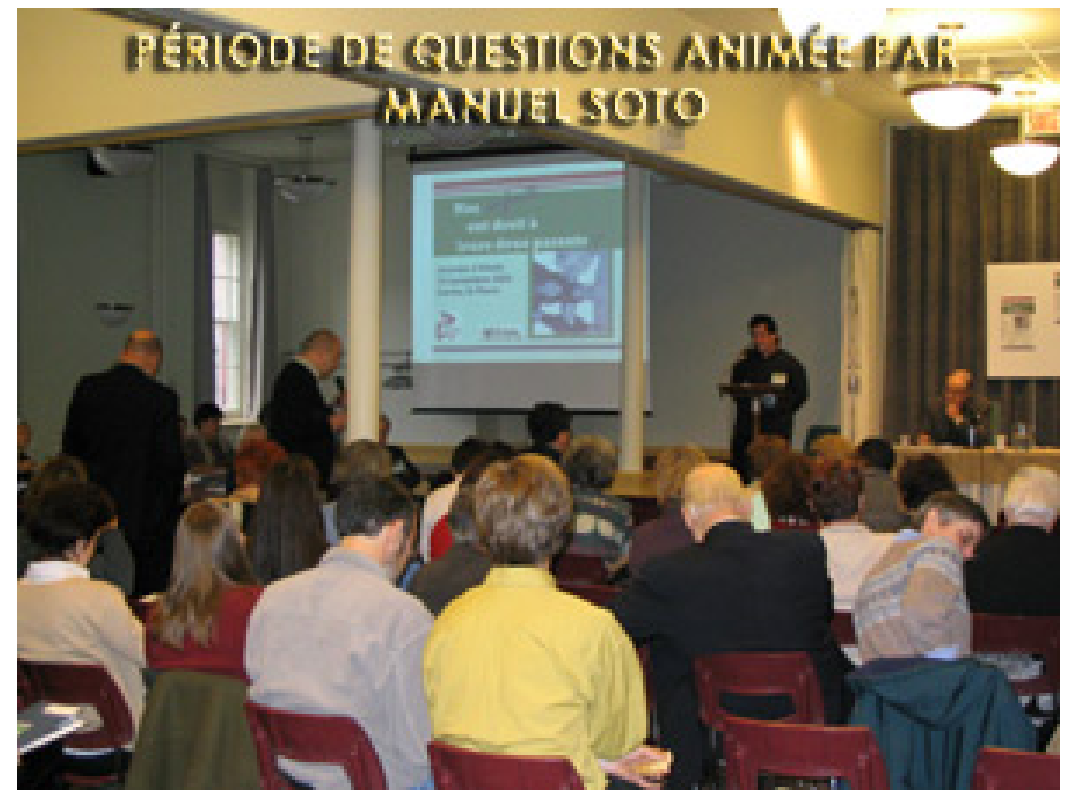

Fin du texte de la conférence 